\title{
Correction: Genetic characterisation of 22q11.2 variations and prevalence in patients with congenital heart disease
}

Hou H, Chen H, Wang X, et al. Genetic characterisation of 22q11.2 variations and prevalence in patients with congenital heart disease. Arch Dis Child 2020;105:367-74.

During production of this paper, two errors were introduced in the numbers of table 1 . The numbers highlighted in red should be 17.3 and 19.2 respectively instead of 1.7 and 1.9.

\begin{tabular}{lcc}
\hline $\begin{array}{l}\text { Table } 1 \text { Phenotypic characteristics of all patients with CHD and the percentage of 22q11.2 deletions } \\
\text { or amplifications in each group }\end{array}$ & $\begin{array}{l}22 q 11.2 \text { positive patients, } \\
\mathbf{n}(\%)\end{array}$ \\
\hline $\begin{array}{l}\text { Phenotypic characteristics } \\
\text { (heart disease) }\end{array}$ & Patients (n) & $15(7.8)$ \\
\hline VSD & 192 & $6(4.3)$ \\
\multicolumn{1}{|c|}{ Isolated VSD } & 140 & $9(17.3)$ \\
\hline VSD with other defects & 52 & $11(12.1)$ \\
ASD & 91 & $6(9.2)$ \\
\hline Isolated ASD & 65 & $5(19.2)$ \\
\hline ASD with other defects & 26 & $12(13.6)$ \\
\hline PDA & 88 & $5(10.2)$ \\
\hline Isolated PDA & 49 & $7(17.9)$ \\
\hline PDA with other defects & 39 & $6(23.1)$ \\
\hline TOF & 26 & $2(28.6)$ \\
\hline DCRV & 7 & $1(25)$ \\
\hline DORV & 4 & $4(15.4)$ \\
\hline RVOTS & 26 & $2(16.7)$ \\
\hline PS & 12 & $18(18)$ \\
\hline PH & 100 & .
\end{tabular}

ASD, atrial septal defect; CHD, congenital heart disease; DCRV, double-chamber right ventricle; DORV, double-outlet right ventricle; PDA, patent ductus arteriosus; PH, pulmonary hypertension; PS, pulmonary stenosis; RVOTS, right ventricular outflow tract stenosis; TOF, tetralogy of Fallot; VSD, ventricular septal defect.

(C) Author(s) (or their employer(s)) 2020. No commercial re-use. See rights and permissions. Published by BMJ. Arch Dis Child 2020;105:e7. doi:10.1136/archdischild-2018-316634corr1

A) Check for updates 